\title{
CONSUMO DE ÁLCOOL POR ENFERMEIROS E IMPLICAÇÕES PARA O TRABALHO EM SAÚDE - REVISÃO INTEGRATIVA
}

\author{
Analy da Silva Machado ${ }^{1}$, Pedro Sadi Monteiro² ${ }^{2}$ Laiane Medeiros Ribeiro ${ }^{3}$, Dirce Guilhem ${ }^{4}$
}

\begin{abstract}
RESUMO: Trata-se de estudo exploratório descritivo com objetivo de descrever o padrão de consumo de álcool entre enfermeiros e suas implicações para o trabalho em saúde. A pesquisa foi realizada em outubro de 2015, em cincobases eletrônicas de dados. Após a aplicação dos critérios de inclusão e exclusão, a amostra final resultou em seis publicações. Os estudos selecionados mostraram que o consumo de bebidas alcoólicas por profissionais de enfermagemestava presente, de forma preocupante, em todas as pesquisas analisadas. Os resultados mostraram que há prevalência de profissionais de enfermagem com problemas relacionados ao consumo abusivo de álcool, em alguns menos e em outros mais acentuados. Observou-se que o trabalho noturno, longas jornadas de trabalho e carga horária semanal elevada demonstraram relação positiva com o consumo de álcool. Além disso, estresse, depressão, ansiedade e insatisfação com o trabalho foram apontados como fatores de risco para o abuso de álcool nos estudos.

DESCRITORES: Enfermeiras e enfermeiros; Transtornos relacionados ao uso de álcool; Transtornos induzidos por álcool; Consumo de bebidas alcoólicas.
\end{abstract}

\section{ALCOHOL USE BY NURSES AND ITS EFFECTS ON HEALTH CARE - INTEGRATIVE REVIEW}

\begin{abstract}
Descriptive and exploratory study aimed to describe the patterns of alcohol use among nurses and its effects on healthcare. The study was conducted in October 2015, with the search of five electronic databases. After implementation of the inclusion and exclusion criteria, the final sample consisted of six publications. The selected publications showed that alcohol use by nursing professionals is a matter of concern, since there is a prevalence of nursing professionals with less or more severe problems related to alcohol use. Night work, long working hours and excessive weekly workloads were positively correlated to alcohol use. In addition, stress, depression, anxiety and dissatisfaction with work were reported as risk factors for excessive drinking in the studies. DESCRIPTORS: Female and male nurses; Alcohol-use related disorders; Alcohol-induced disorders; Consumption of alcohol beverages.
\end{abstract}

\section{CONSUMO DE ALCOHOL POR ENFERMEROS Y CÓMO ESO IMPACTA EN EL TRABAJO EN SALUD - REVISIÓN INTEGRATIVA}

RESUMEN: Estudio exploratorio descriptivo cuyo objetivo fue describir el modelo de consumo de alcohol entre enfermeros y sus implicaciones para el trabajo en salud. La investigación fue realizada en octubre de 2015, en cinco bases electrónicas de datos. Despues de la aplicación de los criterios de inclusión y exclusión, la muestra final resultó en seis publicaciones. Los estudios seleccionados mostraron que el consumo de bebidas alcohólicas por profesionales de enfermería fue constante, de modo preocupante, en todas las investigaciones analizadas. Los resultados revelaron prevalencia de profesionales de enfermería con problemas relacionados al consumo abusivo de alcohol, algunos menos y otros más acentuados. Se observó que el trabajo nocturno, largas jornadas de trabajo y carga horaria semanal elevada presentaron relación con el consumo de alcohol. Además, estrés, depresión, ansiedad e insatisfacción con el trabajo fueron apuntados como factores de riesgo para el abuso de alcohol en los estudios.

DESCRIPTORES: Enfermeras y enfermeros; Trastornos relacionados al uso de alcohol; Trastornos inducidos por alcohol; Consumo de bebidas alcohólicas.

${ }^{1}$ Enfermeira. Mestranda em Enfermagem. Departamento de Enfermagem. Universidade de Brasília. Brasília, DF, Brasil.

${ }^{2}$ Enfermeiro. Doutor em Ciências da Saúde. Docente do Programa de Pós-Graduação em Enfermagem. Universidade de Brasília, Brasília, DF, Brasil.

${ }^{3}$ Enfermeira. Doutora em Enfermagem. Docente do Programa de Pós-Graduação em Enfermagem. Universidade de Brasília, Brasília, DF, Brasil.

${ }^{4}$ Enfermeira. Doutora em Ciências da Saúde. Docente do Programa de Pós-Graduação em Enfermagem. Universidade de Brasília. Brasília, DF, Brasil. 


\section{- INTRODUÇÃO}

O consumo de bebidas alcoólicas é um costume presente na maioria das culturas, principalmente em comemorações sociais. Por outro lado, ouso abusivo desta substância constitui fator de risco para doençascrônicas não transmissíveis como dependência, cirrose hepática e câncer. Além disso, o consumo de álcoolestá associado a acidentes e episódios de violência. Esses fatores estão diretamente relacionados a situações que levam àincapacidade e morte, ao redor do mundo ${ }^{(1)}$.

Em 2014, segundo a Organização Mundial de Saúde (OMS), a média de consumo global de álcool de indivíduos acima de 15 anos foi em torno de 6,2 litros de álcool puro por ano. No Brasil, essa média é de 8,7 litros, quantidade que supera consideravelmente a média mundial. Apesar de elevado, este valor indica uma redução no consumo médio de álcool, uma vez que o levantamento havia apontado um consumo de 9,8 litros em $2005^{(1)}$.

O padrão de consumo de álcool ao longo do tempo,associado à quantidade consumida, está diretamente relacionado ao risco para prejuízos. O uso pesado episódico - representado por cerca de cinco doses ou mais (cerca de 60 gramas de álcool puro) em uma única ocasião ao menos uma vez no último mês - está ligado a problemas agudos, como acidentes automobilísticos, brigas de rua e violência doméstica. Esse padrão foi constatado em $16 \%$ da população mundial consumidora de álcool, enquanto esse percentual chegou a 22\% entre os brasileiros, em $2010^{(1)}$.

A Pesquisa Nacional de Saúde (PNS) de 2013, publicada pelo Instituto Brasileiro de Geografia e Estatística (IBGE), indicou que a proporção de indivíduos com 18 anos ou mais que tinham o costume de consumir bebidas alcoólicas uma vez ou mais por semana era de $24 \%$. Outra consideração importante diz respeito ao grau de escolaridade, pois se percebe que adultos com maior escolaridade apresentavam prevalência de consumo de álcool 30,5\% maior do que aqueles adultos com nível de instrução menor ${ }^{(2)}$.

Em relação à mortalidade,estima-se que 3,3 milhões de mortes no mundo estão relacionadas direta ou indiretamente ao consumo de álcool, o que corresponde a $6 \%$ de todas as mortes ao redor do globo $^{(1)}$.

Estudorealizado em hospital do Rio de Janeiro, em 2014, que teve como objetivo descrever padrões de consumo de álcool, com repercussão na saúde da equipe de enfermagem, mostrou que $67,7 \%$ dos participantes afirmaram ter consumido bebidas alcoólicas, nos últimos 12 meses. Além disso, 21,1\% dos entrevistados haviam bebido, pelo menos, uma vez por semana no último ano. Destes, 6,6\% afirmaram consumir álcool de duas a três vezes por semana ${ }^{(3)}$. Foi identificado, ainda, que 2,2\% da população do estudo consumia álcool acima de três vezes por semana. Resultado importante desse estudo indicou que $26,6 \%$ dos entrevistados associaram o consumo de bebidas alcoólicas com insatisfação no trabalho, estresse, problemas financeiros e conflitos com chefias e colegas $^{(3)}$.

Outro estudo realizado em 2011, com enfermeiras que trabalhavam em hospitais públicos chineses, correlacionou o consumo de álcool como fator de risco para o desenvolvimento de quadro depressivo naquela população ${ }^{(4)}$.

Pesquisas relacionadas ao padrão de consumo de bebidas alcoólicas são mais frequentes entre estudantes de enfermagem, mas poucos são os trabalhos focados em profissionais de enfermagem.

Esta revisão integrativa emergiu da necessidade de investigação sobre o consumo de álcool na vida dos enfermeiros, de modo a identificar o padrão de consumo entre esses profissionais, bem como se os fatores estressores relacionados ao trabalho levaram ao consumo de álcool. O tema desta revisão foi proposto pensando-se na seguinte questão norteadora: Qual o comportamento dos enfermeiros em relação ao consumo de bebidas alcoólicas?

\section{MÉTODO}

Trata-se de estudo exploratório, descritivo, de revisão integrativa e que se refere a um método de pesquisa que permite a incorporação de evidências à prática clínica. Para o desenvolvimento 
deste trabalho, seguiram-se seis etapas: 1 - Identificação do tema e seleção da hipótese ou questão de pesquisa para a elaboração da revisão integrativa; 2) Estabelecimento de critérios para inclusão e exclusão de estudos e busca na literatura; 3) Definição das informações a serem extraídas dos estudos selecionados/categorização dos estudos; 4) Avaliação dos estudos incluídos na revisão integrativa; 5) Interpretação dos resultados; 6) Apresentação da revisão/síntese do conhecimento(5).

Na primeira etapa, o tema desta revisãofoi pensadopara avaliar o padrão de consumo de álcool entre enfermeiros, bem como sua relação com fatores estressores relacionados ao trabalho.

Na segunda etapa,utilizaram-se seis recursos eletrônicos, sendo cinco bases de dados eletrônicas: Literatura Latino-Americana e do Caribe em Ciências da Saúde (LILACS), Cummulative Index to Nursing and Allied Health Literature (CINAHL), Base de Dados de Enfermagem (BDENF), Web of Science e Biblioteca Virtual em Saúde (BVS) - além do portal Pubmed, que engloba o Medical Literature Analysis and Retrievel System Online (MEDLINE), mantidos pela US National Library of Medicine.

Os critérios de inclusão dos artigos foram os seguintes: artigos originais, publicados entre os anos de 2011 a 2015, disponíveis na íntegra, na língua inglesa, portuguesa e espanhola, que traziam em seu conteúdo dados sobre consumo de álcool por enfermeiros.Foram excluídos artigos de revisão, informes ou teses. A pesquisa foi realizada em outubro de 2015.

Em todas as bases, após a busca inicial, foram aplicados os filtros para artigos em língua portuguesa, inglesa e espanhola, publicados entre 2011 e 2015 e disponíveis online na íntegra.

A terceira etapa consistiu na busca das informações a serem extraídas dos estudos, como prevalência dos consumidores, quantidade ingerida, frequência de consumo e possíveis problemas de saúde e sociais relacionados ao consumo de álcool por enfermeiros.

As etapas quatro e cinco compreenderam a leitura criteriosa dos estudos e a extração das informações referentes ao consumo de álcool que fossem relevantes para responder às questões de estudo.

A etapa seis consistiu em verificar quais os temas recorrentes apontados pelos estudos selecionados e que atuavam como fatores de risco para o abuso de álcool entre enfermeiros, conforme consta no Quadro 1. Esses temas foram, posteriormente, alvo de ampla reflexão.

\section{RESUlTADOS}

No portal Pubmed, foram utilizados os descritores controlados do Medical Subject Headings $(\mathrm{MESH}) \mathrm{com}$ a seguinte estratégia de busca: (Alcoholic Beveragesor Alcohol-Induced Disorders or Alcohol-Related Disorders or Alcohol Drinking) and (Nurses or Nurse Practitioners or Nurse Midwives), resultando em 1.306 publicações. Após aplicação dos filtros, restaram 89 trabalhos, dos quais quatro foram selecionados por título e resumo.

Nas bases Web of Science e CINAHL,não existem descritores controlados. Na Web of Science, foi usada a estratégia de busca Alcohol AND Nurses que resultou em 828 referências. Após aplicação dos filtros, restaram 149, das quais quatro foram selecionadas. No CINAHL, foi adotada a mesma estratégia de busca utilizada no Pubmed, o que permitiu encontrar 535 artigos. Após aplicação dos filtros, restaram 90 publicações, das quais quatro foram selecionadas por título e resumo.

Nas bases de dados BVS, LILACS e BDENF, foram utilizados os Descritores em Ciências da Saúde - DeCs com a estratégia (Alcohol-Related Disorders OR Alcohol-Induced Disorders OR Alcohol Drinking) AND (Nurse Midwives OR Nurse Practitioners OR Nurses). Na BVS, foram encontrados 51 estudos, restando sete após aplicação dos filtros, sendo que nenhum desses foi selecionado por título e resumo. Na LILACS, apenas um resultado foi encontrado, sendo que não foi selecionado por título e resumo. Na BDENF, a pesquisa não retornou nenhuma referência.

As informações referentes aos artigos selecionados são apresentadas no Quadro 1.

Dos seis artigos selecionados, um foi realizado na China, três na Austrália, um na Noruega e um no Reino Unido. Dois publicados em 2011, dois, em 2012 e três, em 2015. Todos os artigos selecionados foram publicados em língua inglesa. 


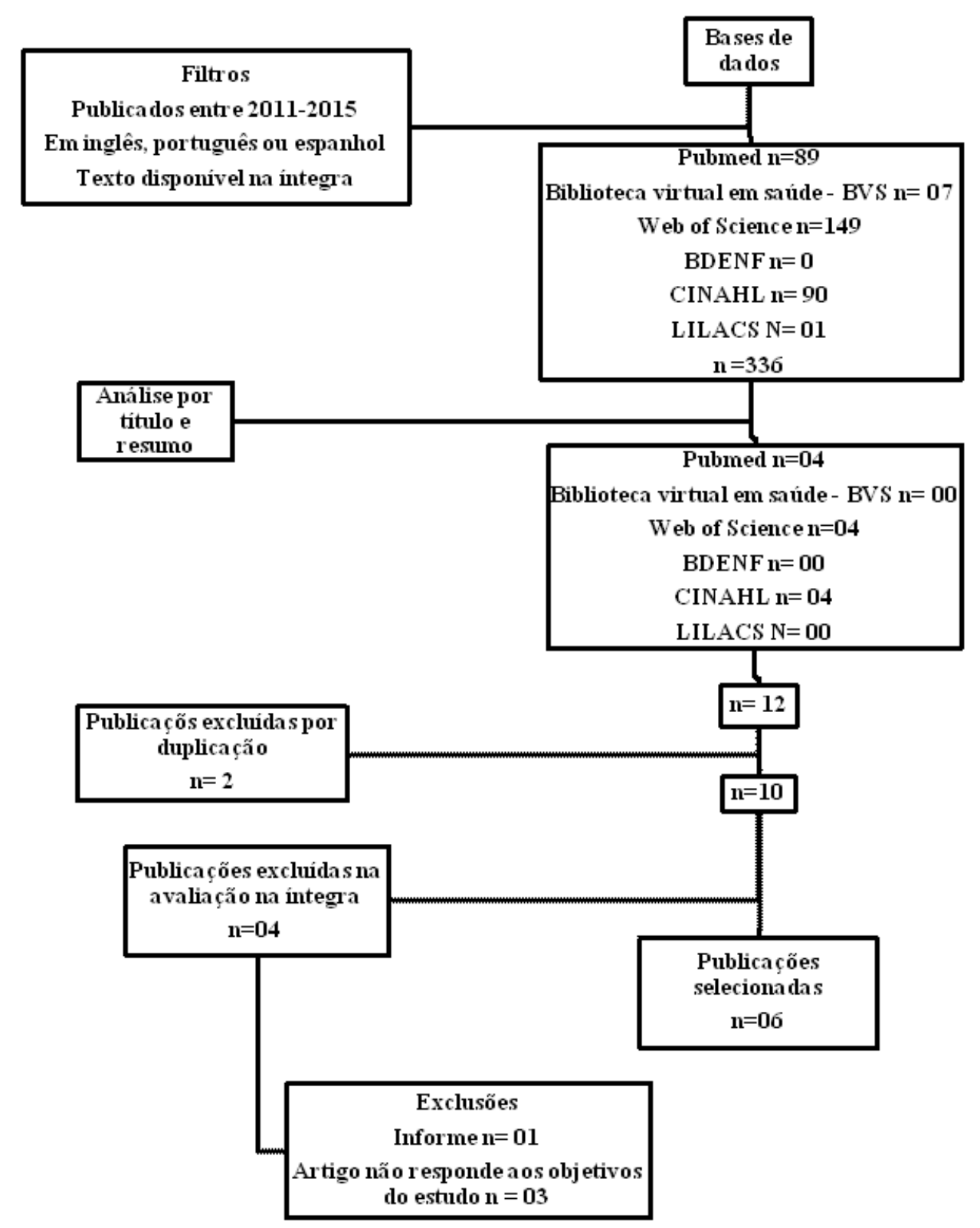

Figura 1 - Estratégia de seleção de artigos. Brasília, DF, Brasil, 2016

Quadro 1 - Apresentação das publicações selecionadas e que fizeram parte da análise. Brasília, DF, Brasil, 2016 (continua)

\begin{tabular}{|l|l|l|}
\hline Autor/Ano & Thtulo do artigo & Tipo de estudo / Resultados \\
\hline Cheung T, Yip PS. 2015 & $\begin{array}{l}\text { Depression, anxiety and } \\
\text { symptoms of stress among } \\
\text { Hong Kong nurses: across- } \\
\text { sectional study. }\end{array}$ & $\begin{array}{l}\text { Estudo transversal. Mostrou que enfermeiras são mais } \\
\text { depressivas, ansiosas e estressadas do que a população } \\
\text { local em geral. Cerca de um terço dos participantes foi } \\
\text { classificado com alguma destas desordens. }\end{array}$ \\
\hline $\begin{array}{l}\text { Perry L, Gallagher R, } \\
\text { Duffield C. 2015 }\end{array}$ & $\begin{array}{l}\text { The mental health of nurses } \\
\text { in acute teaching hospital } \\
\text { settings: a cross-sectional } \\
\text { survey. }\end{array}$ & $\begin{array}{l}\text { Estudo transversal. Demonstrou que é necessário } \\
\text { que enfermeiros e empregadores se empenhem para } \\
\text { promover a saúde o bem-estar no local de trabalho, } \\
\text { pois a ausência destes fatores pode ocasionar } \\
\text { situações deletérias para a saúde mental da equipe. }\end{array}$ \\
\hline $\begin{array}{l}\text { Bakhshi S, Sun F, } \\
\text { Murrells T, While A. } \\
\text { 2015 }\end{array}$ & $\begin{array}{l}\text { Nurses' health behaviours } \\
\text { and physical activity-related } \\
\text { health-promotion practices. }\end{array}$ & $\begin{array}{l}\text { Estudo transversal. Apontou a necessidade de } \\
\text { conscientizar os enfermeiros para a prática de } \\
\text { atividades físicas. Mostrou que as empresas devem } \\
\text { se envolver no sentido de promover a prática de } \\
\text { atividades físicas na prática clínica destes profissionais. }\end{array}$ \\
\hline
\end{tabular}




\begin{tabular}{|c|c|c|}
\hline $\begin{array}{l}\text { Flo E, Pallesen S, } \\
\text { Magerøy N, Moen BE, } \\
\text { Grønli J, Hilde Nordhus } \\
\text { I, Bjorvatn B. } 2012\end{array}$ & $\begin{array}{l}\text { Shift work disorder in nurses } \\
\text { - assessment, prevalence } \\
\text { and related health problems. }\end{array}$ & $\begin{array}{l}\text { Estudo transversal. Indicou que a prevalência de } \\
\text { sintomas indicativos de transtornos de trabalho por } \\
\text { turnos foi alta. }\end{array}$ \\
\hline $\begin{array}{l}\text { Schluter PJ, Turner C, } \\
\text { Benefer C. } 2012\end{array}$ & $\begin{array}{l}\text { Long working hours and } \\
\text { alcohol risk among Australian } \\
\text { and New Zealand nurses and } \\
\text { midwives: A cross-sectional } \\
\text { study. }\end{array}$ & $\begin{array}{l}\text { Estudo transversal. Estabeleceu a relação entre o } \\
\text { consumo de bebidas com longas jornadas de trabalho. } \\
\text { Bem como a necessidade de políticas e programas de } \\
\text { controle destas jornadas de trabalho, afim de garantir } \\
\text { a saúde dos profissionais e dos pacientes com os quais } \\
\text { estes profissionais trabalham. }\end{array}$ \\
\hline $\begin{array}{l}\text { Dorrian J, Paterson J, } \\
\text { Dawson D, Pincombe } \\
\text { J, Grech C, Rogers AE. } \\
2011\end{array}$ & $\begin{array}{l}\text { Sleep, stress and } \\
\text { compensatory behaviors } \\
\text { in Australian nurses and } \\
\text { midwives. }\end{array}$ & $\begin{array}{l}\text { Estudo de coorte. Foi observado que a população } \\
\text { de estudo apresentava uma prevalência elevada de } \\
\text { problemas com o sono, estresse, exaustão e baixa } \\
\text { satisfação profissional. Mostrou também que estes } \\
\text { profissionais lançam mão do uso de álcool e remédios } \\
\text { para indução do sono e consumo de cafeína para } \\
\text { manter o padrão alerta, durante os turnos de trabalho. }\end{array}$ \\
\hline
\end{tabular}

\section{- DISCUSSÃO}

O teste Alcohol Use Disorders Identification Test (AUDIT) - elaborado pela OMS em 1989 - é um instrumento validado e adotado mundialmente, traduzido e adaptado em vários idiomas, para a avaliação do padrão do consumo de álcool. Esse teste busca rastrear o uso excessivo de bebidas alcoólicas,com o intuito de identificar os indivíduos que poderiam se beneficiar com a redução ou cessação do consumo da substância, no nível de atenção primária à saúde.Consiste em um questionário simples de 10 questões que pontua o consumo de álcool entre zero e 40 pontos, identificando quatro zonas de risco: uso de baixo risco (zero a 7 pontos), uso de risco (8 a 15 pontos), uso nocivo (16 a 19 pontos) e provável dependência (20 ou mais pontos) ${ }^{(6)}$.

Diante da simplicidade do instrumento, o AUDIT pode ser adotado em serviços de atenção primária e em pesquisas para identificar potenciais casos que se encontram nas zonas de risco, associados ao uso nocivo ou provável dependência e que, portanto, precisam de intervenções imediatas. Duas pesquisas incluídas nesta revisão integrativa utilizaram essa estratégia para avaliar o consumo de álcool ${ }^{(6)}$.

Estudo sobre a relação entre trabalho noturno e problemas de saúde de enfermeiros, realizado na Noruega, analisou consumo de álcool por meio do teste AUDIT. Apesar de ter sido detectado que havia profissionais com escore de riscopara consumo de álcool, o trabalho não permitiu estabelecer uma relação precisa entre esse consumo e o transtorno investigado ${ }^{(7)}$.

No Reino Unido, foi realizado estudo transversal com o objetivo de determinar os comportamentos de saúde dos enfermeiros e as práticas de promoção da saúde relacionadas à atividade física. Foram avaliados padrões de atividade física, consumo de álcool, hábito de fumar e peso. Para o consumo de álcool, foram utilizadas as três primeiras perguntas do AUDIT: Com que frequência você toma bebidas alcoólicas? Nas ocasiões em que bebe, quantas doses você costuma tomar? Com que frequência você toma "seis ou mais doses" em uma ocasião? Foram entrevistados 623 enfermeiros, sendo que 89,5\% eram do sexo feminino. Em relação ao consumo de álcool, 22\% $(n=133)$ dos entrevistados afirmaram nunca ter consumido álcool ${ }^{(8)}$.

Por outro lado, dentre os $78 \%$ que faziam uso da substância, $25 \%(n=144)$ estavam em situação de risco, isto é, acima de oito pontos conforme a escala do teste AUDIT ${ }^{(8)}$. Desses, os que apresentavam alto risco para consumo de álcool - ou seja, aqueles que alegaram consumir álcool quatro vezes ou mais por semana - correspondiam a um quinto, equivalente a 29 entrevistados. Já os de baixo risco, até sete pontos no AUDIT, representavam 1\% $(n=6)$ da amostra total. A maioria da população de estudo, 88\% ( $n=511)$, afirmou consumir 1-4 doses contendo álcool, num típico dia em que bebiam ${ }^{(8)}$.

Outro estudo, desta vez realizado no Brasil, investigou o consumo de drogas por estudantes de pós-graduação em cursos das ciências da saúde, sendo que grande parte da população de estudo era 
constituída por enfermeiros. Quando questionados sobre a experimentação ou uso de drogas alguma vez na vida, $83 \%$ dos participantes responderam positivamente, sendo a maior proporção relacionadaà bebida alcoólica (51,4\%). Esse trabalho utilizou o AUDIT para avaliar o padrão de consumo de álcool e evidenciou que $30,6 \%$ dos consumidores dessa substância estavam na faixa de consumo considerada de risco, o que representa um valor um pouco mais elevado do que o estudo anterior ${ }^{(9)}$.

Os demais artigos selecionados não utilizaram um instrumento específico para avaliar o consumo de álcool por enfermeiros, porém verificaram se esses profissionais consumiam esta substância em questionamentos que faziam parte do instrumento de coleta de dados de cada estudo.

Nesse sentido, cita-se o estudo realizado com 41 enfermeiras e 21 parteiras de hospitais australianos, no período de 2005 a 2009, que tinha como objetivo descrever o sono, o estresse e os comportamentos compensatórios entre essas profissionais. A pesquisa identificou que $44 \%$ das enfermeiras e $9 \%$ das parteiras relataram o uso de álcool como auxílio para dormir por, pelo menos, uma vez durante a realização do estudo. Ademais, constatou-se que um em cada três participantes do estudo consumia bebidas alcoólicas regularmente como estratégia para induzir o sono. No entanto, não foram coletados detalhes como quantidade de álcool e tipo de bebida alcoólica ingerida, não sendo possível avaliar se o nível de consumo dessa substância seria nocivo para a saúde das participantes ${ }^{(10)}$.

Outra pesquisa que permite identificar o consumo de álcool por enfermeiros trata do estudo transversal realizado na Austrália e Nova Zelândia que buscava analisar a relação entre longas jornadas de trabalho e problemas relacionados ao consumo da substância. Foram incluídos 4.419 enfermeiros e parteiras na amostra, dos quais mais de $90 \%$ eram do sexo feminino, com idades predominando entre 40-49 anos $^{(11)}$.

Dentre os entrevistados, 13,9\% referiram consumo de duas doses ou mais de bebidas alcoólicas por dia e 1,6\% relatou já ter sido diagnosticado com problema relacionado à dependência do álcool. Outra observação importante esteve ligada ao fato de que $0,3 \%$ indicou o consumo diário de bebida alcoólica acima de duas doses e diagnóstico para problemas com o álcool, enquanto 13,6\%, apesar de consumir mais que duas doses diárias, não tinham indicação de diagnóstico para problemas com álcool $^{(11)}$.

Em contrapartida, 1,2\% da população do estudo, apesar de consumir menos que duas doses diárias de bebida, foi diagnosticada com problemas relacionados ao álcool. Por fim, 84,9\% dos participantes consumiam menos que duas doses diárias e não possuíam problemas relacionados ao consumo da substância ${ }^{(11)}$.

Outra observação importante nesse estudofoi que à medida que os profissionais tinham maior média de carga horária semanal, maior era a porcentagem do consumo de bebidas alcoólicas acima de duas doses. Para jornadas semanais médias de até 30 horas, este consumo estava presente em 11,8\% dos participantes; entre 30-39 horas, o percentual era de 13,8\%; entre 40-49 horas, 15,4\%; e acima de 50 horas, $20,2 \%$. Esses dados corroboram os resultados de um trabalho realizado com enfermeiras norueguesas que sugeriu correlação positiva entre carga horária semanal elevada e maior consumo de álcool ${ }^{(11-12)}$.

Similarmente, oestudo transversalque examina a prevalência de casos de depressão, ansiedade e estresse entre os enfermeiros que atuavam em serviços de saúde de Hong Kong apresenta dados sobre a temática. A amostra de estudo era composta por 850 enfermeiros registrados na Associação de Hong Kong de Pessoal de Enfermagem (AHKNS), dos quais 87,6\% eram do sexo feminino, com idade média entre 34-44 anos de idade e 10 anos de experiência clínica ${ }^{(13)}$.

Dentre as características avaliadas, observou-se que $24 \%$ dos entrevistados consumiam álcool, e que esse consumo apresentava relação significativa com o estresse. Dos participantes que relataram o consumo de bebidas alcoólicas, $20 \%$ consumiam álcool não mais que duas vezes ao mês, sendo que esse grupo possuía maior propensão para relatar sintomas de estresse do que aqueles que não bebiam ou que bebiam habitualmente (diariamente ou algumas vezes por semana) ${ }^{(13)}$.

Os autores chegaram a três possíveis explicações para esse achado. O primeiro estaria relacionado ao fato de que osenfermeiros estressados bebem para relaxar, o estudo mostrou ainda que a maioria deles era sedentária. Segundo, notou-se que muitos enfermeiros sofriam de doenças físicas e 
psiquiátricas crônicas, o que comprometia a adoção de um estilo de vida saudável. Desta forma, podese dizer que os enfermeiros bebem como ajuda para a superação do estresse e para a indução do sono. Esse último corrobora o estudo anteriormente citado, no qual se verificou que o consumo de drogas está relacionado à situações de stress e más condições do ambiente de trabalho ${ }^{(9,13)}$.

Por fim, cita-se o estudo exploratório realizado na Austrália, cujos objetivos foram investigar a saúde geral dos enfermeiros e a presença de doença crônica, descrever comportamentos relacionados à saúde destes profissionais e compará-los aos da população em geral. A amostra abrangeu 381 enfermeiros, com idade média de 39,9 anos, sendo que $82,7 \%$ eram do sexo feminino, $80 \%$ trabalhavam em tempo integral e $93 \%$ trabalhavam em regime de $\operatorname{turnos}^{(14)}$.

Foi evidenciado que $92,5 \%$ dos participantes relataram consumir bebidas alcoólicas, dos quais 3,5\% ingeriam a substância diariamente. Além disso, destaca-se que 39,9\% dos participantes que consumiam bebidas alcoólicas foram identificados dentro do grupo de alto risco ${ }^{(14)}$.

A ingestão de álcool é uma condição preocupante também nos demais profissionais de saúde. Conforme estudo realizado na Alemanha com 790 médicos clínicos, constatou-se que 23\% desses relataram consumo diário de algum tipo de bebida alcoólica ${ }^{(15)}$.

Outro trabalho nesta mesma linha de pesquisa, desta vez realizado na Noruega, analisou a variação no padrão de consumo de álcool por médicos no período de dez anos. Observou-se que entre os anos 2.000 e 2.010 houve um aumento de $31,4 \%$ para $48,7 \%$ dos médicos que usaram álcool duas vezes ou mais por semana,enquanto os consumidores considerados de risco diminuíram de 10,7\% para 8,2\% no mesmo período. Uma das questões apontadas pelo estudo, acerca da diminuição da população com padrão de risco para consumo de álcool, é a tendência mundial para adoção de um estilo de vida mais saudável, pois a diminuição do consumo desta substância é fator importante para essa nova realidade ${ }^{(16)}$.

\section{CONSIDERAÇÕES FINAIS}

A partir das referências encontradas,destaca-se que o estudo sobre o consumo de álcool por enfermeiros é ainda pouco explorado, observando-se uma concentração dos estudos em determinadas áreas geográficas. A busca não resgatou nenhum estudo realizado no Brasil com a temática proposta. Até mesmo os estudos incluídos nessa revisão não tinham como objetivo principal o consumo de álcool, por parte de profissionais de enfermagem.

Com base nos estudos selecionados, buscou-se estabelecer o padrão de consumo de bebidas alcoólicas, a fim de relacioná-lo com outras hipóteses dos estudos. Os resultados mostraram que há prevalência de profissionais de enfermagem com problemas relacionados ao consumo abusivo de álcool, em alguns menos e em outros mais acentuados.

A relação com trabalho noturno é importante, pois os estudos mostraram que muitos profissionais que atuam nesses períodos muitas vezes consomem álcool, a fim de induzir o sono ou de relaxar. As longas jornadas de trabalho e a carga horária semanal total (incluindo aqueles que possuem mais de um emprego) devem ser avaliadas cautelosamente, uma vez que houve relação entre a duração da jornada de trabalho com o consumo de álcool.

O estresse, depressão, ansiedade e insatisfação com o trabalho foram pontos abordados nos estudos e são vistos como fatores de risco para abuso de álcool.

Estudos adicionais são necessários para explorar mais adequadamente esta temática, a fim de estabelecer detalhadamente estes padrões.

\section{- REFERÊNCIAS}

1. World Health Organization (WHO). Global status report on alcohol and health - 2014. [Internet] 2014 [acesso em 22 nov 2015]. Disponível: http://www.who.int/substance_abuse/publications/global_alcohol_report/en/. 
2. Instituto Brasileiro de Geografia e Estatística (IBGE). Pesquisa Nacional de Saúde: 2013: acesso e utilização dos serviços de saúde, acidentes e violências : Brasil, grandes regiões e unidades da federação / IBGE, Coordenação de Trabalho e Rendimento. Rio de Janeiro: IBGE; 2015.

3. de Oliveira EB, Fabri JMG, de Paula GS, de Souza SRC, da Silveira WG, Matos GS.Padrões de uso de álcool por trabalhadores de enfermagem e a associação com o trabalho. Rev. enferm. UERJ. [Internet] 2014;21(6) [acesso em 22 nov 2015]. Disponível: http://www.e-publicacoes.uerj.br/index.php/enfermagemuerj/article/view/11514.

4. Gao YQ, Pan BC, Sun W, Wu H, Wang JN, Wang L. Depressive symptoms among Chinese nurses: prevalence and the associated factors. J AdvNurs. [Internet] 2012;68(5)[acesso em 22 nov 2015]. Disponível: http://dx.doi. org/10.1111/j.1365-2648.2011.05832.x.

5. Mendes KDS, Silveira RCCP, Galvão CM. Revisão integrativa: método de pesquisa para a incorporação de evidências na saúde e na enfermagem. Texto Contexto Enferm. [Internet] 2008;17(4) [acesso em 22 nov 2015]. Disponível: http://dx.doi.org/10.1590/S0104-07072008000400018.

6. Jomar RT, da Paixão LAR, Abreu AMM. Alcohol Use DisordersIdentification Test (AUDIT) e sua aplicabilidade na atenção primária à saúde. Rev. APS. [Internet] 2012;15(1) [acesso em 22 nov 2015] Disponível: https://aps.ufjf. emnuvens.com.br/aps/article/view/1467/598.

7. Flo E, Pallesen S, Magerøy N, Moen BE, Grønli J, Nordhus IH, et al.Shift Work Disorder in Nurses - Assessment, Prevalence and Related Health Problems. PLoS ONE. [Internet] 2012;7(4) [acesso em 25 out 2015]. Disponível: http://doi.org/10.1371/journal.pone.0033981.

8. Bakhshi S, Sun F, Murrells T, While A. Nurses' health behaviours and physical activity-related healthpromotion practices. Br J Community Nurs.[Internet] 2015;20(6)[acessoem 25 out 2015]. Disponível: http://dx.doi. org/10.12968/bjcn.2015.20.6.289.

9. da Rocha PR, David HMSL. Patterns of alcohol and drug consumption in health care professionals: a portrait of students of latosensu courses in a public institution.SMAD, Rev. eletrônica saúde mental alcooldrog. [Internet] 2015;11(1) [acesso em 17 jul 2016]. Disponível: http://dx.doi.org/10.11606/issn.1806-6976.v11i1p41-48.

10. Dorrian J, Paterson J, Dawson D, Pincombe J, Grech C, Rogers AE. Sleep, stress and compensatory behaviors in Australian nurses and midwives. Rev. Saúde Pública. [Internet] 2011;45(5)[acesso em 24 out 2015]. Disponível:http:// dx.doi.org/10.1590/S0034-89102011005000059.

11. Schluter PJ, Turner C, Benefer C. Long working hours and alcohol risk among Australian and New Zealand nurses and midwives: A cross-sectional study. Int J Nurs Stud. [Internet] 2012;49(6) [acesso em 24 out 2015]. Disponível: http://dx.doi.org/10.1016/j.ijnurstu.2012.01.005.

12. Buchvold HV,Pallesen S,Oyane NM, Bjorvatn B.Associations between night work and BMI, alcohol, smoking, caffeine and exercise--a cross-sectional study. BMC Public Health.[Internet]2015;(15)[acesso em 17 jul 2016]. Disponível: http://dx.doi.org/10.1186/s12889-015-2470-2.

13. Cheung T, Yip PS. Depression, anxiety and symptoms of stress among hongkong nurses: a cross-sectional study.Int J Environ Res Public Health.[Internet]2015;12(9)[acessoem 24 out 2015]. Disponível: http://dx.doi. org/10.3390/ijerph120911072.

14. Perry L, Gallagher R, Duffield C. The health and health behaviours of Australian metropolitan nurses: an exploratory study.BMC Nurs. [Internet] 2015;(14) [acesso em 24 out 2015]. Disponível: http://bmcnurs. biomedcentral.com/articles/10.1186/s12912-015-0091-9.

15. Unrath M, Zeeb H, Letzel S, Claus M, Escobar Pinzón LC. Identification of possible risk factors for alcohol use disorders among general practitioners in Rhineland-Palatinate, Germany. SwissMedWkly. [Internet] 2012;(142) [acesso em 17 jul 2016]. Disponível: http://dx.doi.org/10.4414/smw.2012.13664.

16. Rosta J, Aasland OG. Changes in alcohol drinking patterns and their consequences among Norwegian doctors from 2000 to 2010: a longitudinal study based on national samples. AlcoholAlcohol.[Internet] 2013;48(1) [acesso em 17 jul 2016]. Disponível: http://dx.doi.org/10.1093/alcalc/ags084. 